\title{
Solar Sources of "Driverless" Interplanetary Shocks
}

\author{
N. Gopalswamy ${ }^{1}$, P. Mäkelä ${ }^{2}$ H. Xie ${ }^{2}$, S. Akiyama ${ }^{2}$ and S.Yashiro ${ }^{3}$ \\ ${ }^{I}$ NASA Goddard Space Flight Center, Greenbelt, MD 20771, USA \\ ${ }^{2}$ The Catholic University of America, Washington DC 20064, USA \\ ${ }^{3}$ Interferometrics, Herndon, VA 20171, USA
}

\begin{abstract}
We identify the solar sources of a large number of interplanetary (IP) shocks that do not have a discernible driver as observed by spacecraft along the Sun-Earth line. At the Sun, these "driverless" shocks are associated with fast and wide CMEs. Most of the CMEs were also driving shocks near the Sun, as evidenced by the association of IP type II radio bursts. Thus, all these shocks are driven by CMEs and they are not blast waves. Normally limb CMEs produce driverless shocks at $1 \mathrm{AU}$. But some disk-center CMEs also result in driverless shocks because of deflection by nearby coronal holes. We estimate the angular deflection to be in the range $20^{\circ}-60^{\circ}$. We also compared the influence of nearby coronal holes on a set of CMEs that resulted in magnetic clouds. The influence is nearly three times larger in the case of driverless shocks, confirming the large deflection required.
\end{abstract}

Keywords: Coronal mass ejections; coronal holes; interplanetary shocks; Type II radio bursts. PACS: 96.50.Ci Solar wind plasma; sources of solar wind; 96.50.Uv Ejecta, driver gases, and magnetic clouds; 96.60.pc Coronal holes; 96.60.ph Coronal mass ejection; 96.50.sh Interplanetary propagation and effects

\section{INTRODUCTION}

It has been known for a long time that some interplanetary shocks are not followed by discernible ejecta [1]. We call these "driverless" shocks. Possible explanations are: (i) the driverless (DL) shocks are caused by coronal mass ejections (CMEs) propagating at large angles to the Sun-Earth line such that an observer near Earth misses the driver [2], and (ii) these are blast waves originating from flare explosions [3]. A recent investigation showed that DL shocks can also originate from near the disk center [4]. Although the disk-center DL shocks are likely candidates for blast waves, we show that they are also associated with fast and wide CMEs, but the CMEs get deflected by nearby coronal holes. In other words, all the DL shocks can be attributed to energetic CMEs at the Sun if one considers the interaction with nearby coronal holes. In this paper, we compare the effect of coronal holes on two sets of CMEs originating close to the disk center, one resulting in DL shocks at $1 \mathrm{AU}$ and the other in magnetic clouds (MCs) driving shocks. In the in-situ data, DL shocks are observed as a discontinuity followed by a sheath region of enhanced proton temperature. On the other hand, shocks driven by MCs are followed by the sheath and a flux-rope structure defined by the three signatures: (i) enhanced magnetic field, (ii) smooth rotation of the magnetic field component perpendicular to the Sun-Earth line, and (iii) low proton temperature compared to the solar wind [5]. By comparing the disk center and limb sources of DL shocks, we estimate the angle by which CMEs need to be deflected in order for the driver not to be intercepted at $1 \mathrm{AU}$. We also examine the type II burst association, which indicates whether the shocks are already formed near the Sun.

\section{DRIVERLESS SHOCKS OF CYCLE 23}

A total of 225 interplanetary (IP) shocks were detected near Earth by one or more of the spacecraft in the solar wind (SOHO, ACE, and Wind) that had overlapping CME observations from SOHO. A significant number (41 out of 225 or $18 \%$ ) of the IP shocks were not followed by a discernible driver. The solar sources of the IP shocks were identified as whitelight CMEs observed by the Solar and Heliospheric Observatory (SOHO) coronagraphs. Details of the source identifications can be found in [6]. Figure 1 shows the solar source locations of 35 DL shocks. The solar sources of the remaining 6 shocks were behind the limb, so they are not plotted. Shocks with type II bursts (radio loud, RL) and those without (radio quiet, RQ) are distinguished by different symbols. Most of the shocks were RL ( 27 out of 35 or $78 \%$ ), confirming 
that most of the CMEs were driving shocks near the Sun. However, there is no significant difference between the source distributions of RQ and RL shocks.

The DL shocks fall into two groups: the disk center sources (longitudinal distance from the central meridian $\leq 15^{\circ}$ ), and limb sources (longitudinal distance from the central meridian $>45^{\circ}$ ). There are only a few sources at intermediate longitudes. The limb location of the CMEs associated with DL shocks is normal, because the shock flanks arrive at Earth, while the driving CMEs are missed by spacecraft along the Sun-Earth line. On the other hand, the diskcenter location is anomalous because the driving CMEs are expected to arrive at Earth, but they do not. The anomaly has been shown to be due to the presence of coronal holes near the eruption regions [4] such that the CMEs are deflected away from the Sun-Earth line effectively making them behave like limb CMEs. In Fig.1, there are five disk-center DL shocks, which we study here. The marginally disk-center event at E22 has been reported elsewhere [4].

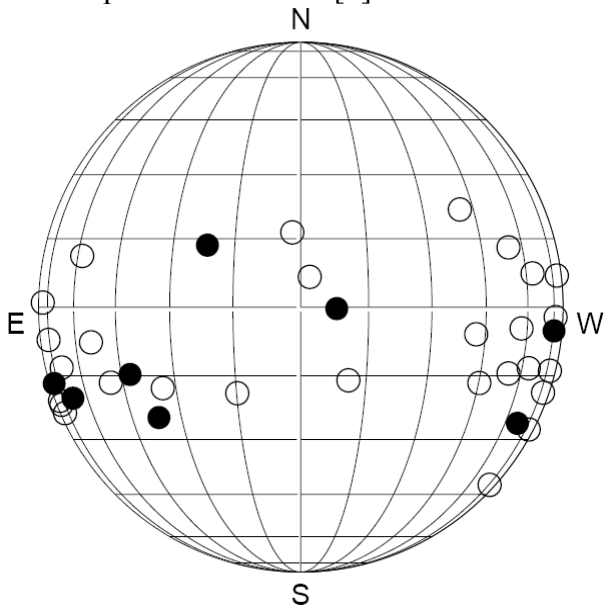

FIGURE 1. Solar sources of 35 DL shocks detected during solar cycle 23. Open (filled) circles denote shocks with (without) type II radio bursts.

\section{Illustrative Example}

Figure 2 shows the active region (AR) 10588 that produced a halo $\mathrm{CME}$ when it was at S18E15. Even though the $\mathrm{CME}$ originated within a central meridian distance (CMD) of $30^{\circ}$ degrees, it produced only a shock at 1 AU. A large coronal hole is located to the north of the active region. The unipolar magnetic field within the coronal hole appears to be a continuation of the negative polarity of the active region. Since the northern leg of the flux rope erupting from such a region has negative polarity (same as that of the coronal hole), no reconnection is expected between the $\mathrm{CME}$ and the coronal hole field lines. Since the CME- driven shock and the flux rope cannot propagate into the coronal hole, the CME gets deflected to the east and south such that the flux rope propagates at a larger angle to the Sun-Earth line, thus missing the observing spacecraft at 1 AU. However, the CME-driven shock is extended enough to be intercepted by the spacecraft, thus appearing as a DL shock. In other words, a CME from S18E15 behaves like a limb CME.

\section{CORONAL HOLE INFLUENCE PARAMETER}

We define the coronal hole influence parameter (CHIP) as the magnitude of a fictitious force (F) that depends on the average photospheric magnetic field (B) in the coronal hole, the size of the coronal hole measured as the dark area (A) observed in SOHO EUV images, and the distance (r) between the centroid of the coronal hole to the eruption center:

$\mathrm{F}=\mathrm{B}^{2} \mathrm{~A} / \mathrm{r}^{2}$.

The distance $\mathrm{r}$ is determined as the straight-line distance between the coronal hole centroid and the eruption center in the sky-plane projection. The unit of $F$ is $G^{2}$. The direction of $F$ is from the coronal-hole centroid to the eruption center. When multiple coronal holes are present, F is computed as the vector sum of the forces exerted by all the coronal holes. In a previous study, $\mathrm{F}$ was taken to be proportional to $\mathrm{B}$ [4]. Here we consider $\mathrm{B}^{2}$ which represents the magnetic pressure. For the example given in Fig. 2, $\mathrm{F}=26 \mathrm{G}^{2}$ and was directed along position angle (PA) $137^{\circ}$. On this day, there was one other smaller coronal hole in the south, which affected very little because $F$ was only $\sim 0.75 \mathrm{G}^{2}$.

TABLE 1. Driverless Shocks From the Disk Center

\begin{tabular}{cllllll}
\hline $\begin{array}{c}\text { CME } \\
\text { Date }\end{array}$ & $\begin{array}{c}\text { CME } \\
\text { Loc }\end{array}$ & $\begin{array}{c}\text { CH } \\
\text { Loc }\end{array}$ & A & r & $\begin{array}{l}<\text { B }> \\
\text { G }\end{array}$ & $\begin{array}{l}\text { F } \\
\mathbf{G}^{2}\end{array}$ \\
\hline $2003 / 04 / 21$ & N08E02 & S06E30 & 4.5 & 4.4 & -18. & 74 \\
$2003 / 11 / 20$ & N01W08 & N06E10 & 7.5 & 2.9 & -8.0 & 63 \\
$2004 / 04 / 06$ & S18E15 & N08W10 & 14 & 4.3 & -5.9 & 26 \\
$2004 / 04 / 08$ & S15W11 & S02W25 & 6.1 & 2.3 & -10. & 120 \\
$2004 / 12 / 03$ & N08W02 & S32E02 & 12 & 4.8 & 5.9 & 18 \\
\hline
\end{tabular}

TABLE 2. Magnetic Cloud Shocks From the Disk Center.

\begin{tabular}{ccccccc}
\hline $\begin{array}{c}\text { CME } \\
\text { Date }\end{array}$ & $\begin{array}{c}\text { CME } \\
\text { Loc }\end{array}$ & $\begin{array}{c}\text { CH } \\
\text { Loc }\end{array}$ & A & r & $\begin{array}{c}\text { B } \\
\text { G }\end{array}$ & $\begin{array}{l}\text { F } \\
\mathbf{G}^{2}\end{array}$ \\
\hline $2002 / 07 / 29$ & S10W10 & N45E21 & 38 & 7.2 & -5.9 & 26 \\
$2002 / 07 / 29$ & N12W16 & N27E35 & 12 & 5.9 & -9.5 & 37 \\
$2003 / 10 / 28$ & S20E02 & N28W35 & 9.5 & 6.9 & -4.1 & 5.7 \\
$2003 / 10 / 29$ & S19W09 & S16E20 & 3.3 & 3.4 & -16. & 75 \\
$2004 / 11 / 06$ & N09E25 & N19W25 & 6.7 & 3.7 & 5.8 & 14 \\
$2005 / 05 / 13$ & N12E11 & S63W11 & 11 & 8.6 & 3.4 & 3.1 \\
\hline
\end{tabular}




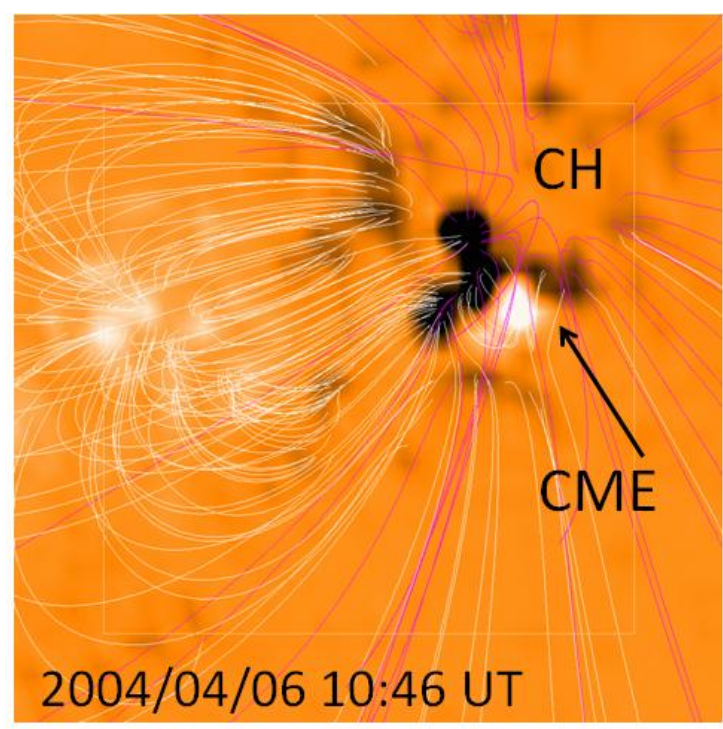

FIGURE 2. Active region (AR 10588 located at S18E15) of the 2004 April $4 \mathrm{CME}$ that resulted in a DL shock at $1 \mathrm{AU}$. A large coronal hole (marked $\mathrm{CH}$ ) is located to the north of the active region. Extrapolated magnetic field lines (white: closed, purple: open) are shown superposed on a SOHO/MDI magnetogram (white: positive, dark: negative). The potential-field rendering of the solar coronal magnetic field corresponding to the pointings of the TRACE spacecraft (the white square) are made available online by $\mathrm{K}$. Schrijver and M. de Rosa at http://www.lmsal.com/forecast/.

\section{Comparison with Magnetic Cloud Shocks}

Tables 1 and 2 list the CMEs (dates and heliographic locations) that resulted in DL shocks and shocks driven by MCs, respectively. Both sets of CMEs originated from close to the disk center. Also listed are the coronal-hole centroids ( $\mathrm{CH} \mathrm{Loc),} \mathrm{area} \mathrm{(A}$ in units of $10^{10} \mathrm{~km}^{2}$ ), the distance ( $\mathrm{r}$ in units of $\mathrm{km}$ ) between the coronal-hole centroid and the eruption region, the average photospheric field $(<\mathrm{B}>)$ within the coronal hole, and the CHIP (F). Only one coronal hole with the largest CHIP is listed for each eruption. The average magnetic field in the coronal holes associated with DL shocks is $\sim 9.54 \mathrm{G}$ compared to 5.8 $\mathrm{G}$ for the coronal holes near MC sources. In addition to the higher $<\mathrm{B}>$, the coronal holes are generally located at shorter distances from the eruption regions in the case of DL shocks. The $\mathrm{F}$ values are accordingly higher: $60.2 \mathrm{G}^{2}$ for DL shock sources vs. $26.6 \mathrm{G}^{2}$ for $\mathrm{MC}$ sources. Clearly, the CHIP is more than two times larger for the CMEs associated with the DL shocks, thus explaining the large deflection of CMEs associated with DL shocks compared to those associated with MCs. The F value computed from $\mathrm{B}^{2}$ shows a slightly better contrast between the DL shocks and the MC-driven shocks. When we repeated the computation of $\mathrm{F}$ for all the IP CMEs (ICMEs) that had a solar source near the disk center, we found an average value of $15.6 \mathrm{G}^{2}$, which is about 4 times smaller than the F for DL shocks.

\section{Deflection Angle}

One can estimate the angle by which the CMEs need to be deflected so that the associated shocks appear driverless at $1 \mathrm{AU}$ based on the source locations of the DL shocks from limb CMEs. The eastern DL shocks (non-disk center) have longitudes ranging from $34^{\circ}$ to $90^{\circ}$, with an average of $65^{\circ} .5$. This suggests that a disk center CME from E15 needs to be deflected by an angle of at least $19^{\circ}$ for it to behave like a limb $\mathrm{CME}$. The deflection could be as high as $50^{\circ}$ to take it to the average location of E65. Similarly the western DL shocks have solar source longitudes in the range $42^{\circ}$ to $90^{\circ}$, with an average value of $68^{\circ} .5$. Therefore, a CME from W11 needs to be deflected by an angle of at least $31^{\circ}$ for it to behave like a limb CME. The deflection angle has to be as high as $57^{\circ} .5$ to take it to the average longitude of the limb CMEs.

\section{Deflection Schematic}

Figure 3 illustrates how CMEs might be deflected by coronal holes. A shock-driving CME heading towards the observer is deflected to the left (away from the Sun-Earth line) by the coronal hole magnetic field located to the right of the eruption region. It is also assumed that the part of the flux rope nearest to the coronal hole and the coronal hole have nearly same field directions, so no reconnection takes place. After deflection, the CME heads at a large angle to the SunEarth line such that it is not intercepted by the observing spacecraft. However, the shock, which is more extended than the $\mathrm{CME}$, is intercepted by the spacecraft, observing it as a DL shock.

\section{DISCUSSION}

The CME deflection needed to make them behave like limb CMEs is estimated to be over a large range: from $19^{\circ}$ to nearly $58^{\circ}$. The actual deflection required probably depends on the orientation of the CME flux rope. For example, a high-inclination flux rope needs to be deflected less in the longitudinal direction compared to a low inclination one because of the smaller east-west extent of the CME. It is also possible that in some cases the ejection may be inherently nonradial in the source active region, especially for CMEs associated with weaker flares. It has been shown that the $\mathrm{CME}$ is radially above the flare site for $\mathrm{X}$ and $\mathrm{M}$ class flares, while it can be offset for weaker flares [7]. 


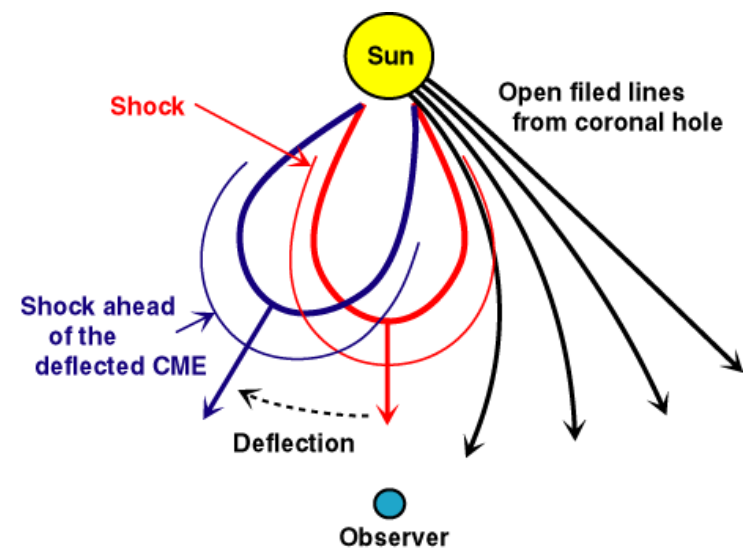

FIGURE 3. A CME initially heading towards the observer is deflected away from the Sun-Earth line by the magnetic field in the coronal hole to the west of the eruption region. The CME and the shock before (after) deflection are drawn in red (blue). The direction of deflection is marked by the dashed arrow. The coronal hole could be anywhere relative to the eruption region and the deflection is away from the coronal hole.

We found that the CHIP shows a better contrast between DL shocks and shocks with ICMEs when it is assumed to be proportional to $\mathrm{B}^{2}$ rather than $\mathrm{B}$ used in [4]. However, the contrast between MCs and noncloud ICMEs is not significant. Therefore, it is not easy to identify deflection as one of the factors that determine the appearance of MCs and non-cloud ICMEs. More detailed analysis is required to address this question.

It must be pointed out that we considered only deflections away from the Sun-earth line. Coronal holes can also deflect CMEs towards the Sun Earth line. If a CME erupting at a larger CMD is deflected towards the Sun-Earth line, it might appear as an ICME at 1 AU. Such a deflection can occur when the coronal hole is at a larger CMD than the eruption region. One example is the 2003 November $18 \mathrm{CME}$, which resulted in the largest geomagnetic storm of solar cycle 23 [8]. A large coronal hole extending in the north-south direction was located immediately to the east of the eruption region, favorable for deflection the CME towards the Sun-Earth line. Interestingly, the same coronal hole deflected another CME from the same region away from the Sun-Earth line two days later because the eruption region rotated to the western hemisphere (2003 November 20 CME in Table 1).

\section{SUMMARY}

The "driverless" interplanetary shocks fall into two groups based on their solar sources of the associated CMEs: the disk-center sources and limb sources. In the case of limb CMEs, the shock flanks arrive at Earth, while the driving CMEs do not. In the case of diskcenter sources, the driving CMEs are expected to arrive at Earth, but they do not. The presence of a coronal hole near the eruption regions seems to make the CMEs behave like limb CMEs. All but one of the disk-center DL shocks occurred during the declining phase, when low-latitude coronal holes occur frequently. The coronal holes were especially prominent during solar cycle 23.

All the DL shocks were associated with fast and wide CMEs near the Sun. Most of the CMEs associated with the DL shocks were also accompanied by type II radio bursts, which means the CMEs were driving shocks near the Sun. Therefore, we can rule out the possibility that they are blast waves. The radio quiet shocks are generally weaker and may be formed at large distances from the Sun. There is no difference in source distributions of radio-quiet and radio-loud shocks. The CMEs associated with the RQ shocks were generally slower and narrower.

The coronal hole influence parameter was nearly three times smaller for the case of MCs driving shocks at $1 \mathrm{AU}$. This means the MC-associated CMEs were not significantly affected by the coronal holes. The influence is smaller because the coronal holes were located at larger distances and contained smaller average magnetic field values. The net result is that CMEs originating from close to the disk center arrived as MCs at 1 AU. This study needs to be extended to other phases of the solar cycle and to other longitudes of CME sources.

\section{ACKNOWLEDGMENTS}

Work supported by NASA's LWS TR\&T program. $\mathrm{SOHO}$ is a project of international collaboration between ESA and NASA.

\section{REFERENCES}

1. R. Schwenn, Astrophys. Space Sci., 243, 187-193 (1996).

2. N. Gopalswamy, A. Lara, M. L. Kaiser, and J.-L. Bougeret, J. Geophys. Res., 106, 25261 - 25278 (2001).

3. S. Y. Oh, Y. Yi, and Y. H. Kim, Solar Phys. 245, 391 410 (2007)

4. N. Gopalswamy, P. Mäkelä, H. Xie, S. Akiyama, and S. Yashiro, J. Geophys. Res., 114, A00A22 (2009).

5. L. Burlaga, E. Sittler, F. Mariani, R. Schwenn, J. Geophys. Res., 86, 6673 (1981).

6. N. Gopalswamy, S. Yashiro, and S. Akiyama, J. Geophys. Res., 112, A06112 (2007).

7. S. Yashiro, G. Michalek, S. Akiyama, N. Gopalswamy, R. A. Howard, Astrophys. J., 673, 1174 -1180 (2008).

8. N. Gopalswamy, S. Yashiro, G. Michalek, H. Xie, R. P. Lepping, and R. A. Howard, Geophysical Res. Lett., 32, L12S09 (2005). 